\title{
A Subfamily of Univalent Functions Associated with $q$-Analogue of Noor Integral Operator
}

\author{
Muhammad Arif, ${ }^{1}$ Miraj U1 Haq, ${ }^{1}$ and Jin-Lin Liu ${ }^{2}{ }^{2}$ \\ ${ }^{1}$ Department of Mathematics, Abdul Wali Khan University Mardan, Mardan, Pakistan \\ ${ }^{2}$ Department of Mathematics, Yangzhou University, Yangzhou 225002, China \\ Correspondence should be addressed to Jin-Lin Liu; jlliu@yzu.edu.cn \\ Received 14 September 2017; Accepted 8 January 2018; Published 1 February 2018 \\ Academic Editor: John R. Akeroyd
}

Copyright (C) 2018 Muhammad Arif et al. This is an open access article distributed under the Creative Commons Attribution License, which permits unrestricted use, distribution, and reproduction in any medium, provided the original work is properly cited.

The main objective of the present paper is to define a new subfamily of analytic functions using subordinations along with the newly defined $q$-Noor integral operator. We investigate a number of useful properties such as coefficient estimates, integral representation, linear combination, weighted and arithmetic means, and radius of starlikeness for this class.

\section{Introduction and Definitions}

In recent years, $q$-analysis ( $q$-calculus) has motivated the researchers a lot due to its numerous applications in mathematics and physics. Jackson $[1,2]$ was the first to give some application of $q$-calculus and also introduced the $q$-analogue of derivative and integral operator. Later on, Aral and Gupta $[3,4]$ defined the $q$-Baskakov-Durrmeyer operator by using $q$-beta function while in papers $[5,6]$ the authors discussed the $q$-generalization of complex operators known as $q$-Picard and $q$-Gauss-Weierstrass singular integral operators. Using convolution of normalized analytic functions, Kanas and Raducanu [7] defined $q$-analogue of Ruscheweyh differential operator and studied some of its properties. The application of this differential operator was further studied by Aldweby and Darus [8] and Mahmood and Sokół [9]. The aim of the current paper is to define a $q$-analogue of the Noor integral operator involving convolution concepts and then give some interesting applications of this operator.

Let us denote the open unit disk by $\mathfrak{D}=\{z \in \mathbb{C}$ : $|z|<1\}$ and the symbol $\mathfrak{A}$ denotes the family of those analytic functions $f$ which has the following Taylor series representation:

$$
f(z)=z+\sum_{k=2}^{\infty} a_{k} z^{k}, \quad(z \in \mathfrak{D}) .
$$

For two functions $f$ and $g$ that are analytic in $\mathfrak{D}$ and have the form (1), we define the convolution of these functions by

$$
f(z) * g(z)=z+\sum_{k=2}^{\infty} a_{k} b_{k} z^{k}, \quad(z \in \mathfrak{D}) .
$$

For $0<q<1$, the $q$-derivative of a function $f \in \mathfrak{A}$ is defined by

$$
\partial_{q} f(z)=\frac{f(q z)-f(z)}{z(q-1)}, \quad(z \neq 0) .
$$

It can easily be seen that for $n \in \mathbb{N}:=\{1,2,3, \ldots\}$ and $z \in \mathfrak{D}$

$$
\partial_{q}\left\{\sum_{n=1}^{\infty} a_{n} z^{n}\right\}=\sum_{n=1}^{\infty}[n, q] a_{n} z^{n-1},
$$

where

$$
[n, q]=\frac{1-q^{n}}{1-q}=1+\sum_{l=1}^{n-1} q^{l}, \quad[0, q]=0 .
$$

For any nonnegative integer $n$, the $q$-number shift factorial is defined by

$$
[n, q] != \begin{cases}1, & n=0, \\ {[1, q][2, q][3, q] \cdots[n, q],} & n \in \mathbb{N} .\end{cases}
$$


Also the $q$-generalized Pochhammer symbol for $x>0$ is given by

$$
[x, q]_{n}= \begin{cases}1, & n=0 \\ {[x, q][x+1, q] \cdots[x+n-1, q],} & n \in \mathbb{N}\end{cases}
$$

For $\mu>-1$, we define the function $\mathscr{F}_{q, \mu+1}^{-1}(z)$ by

$$
\mathscr{F}_{q, \mu+1}^{-1}(z) * \mathscr{F}_{q, \mu+1}(z)=z \partial_{q} f(z),
$$

where the function $\mathscr{F}_{q, \mu+1}(z)$ is given by

$$
\mathscr{F}_{q, \mu+1}(z)=z+\sum_{n=2}^{\infty} \frac{[\mu+1, q]_{n-1}}{[n-1, q] !} z^{n}, \quad(z \in \mathfrak{D}) .
$$

It is quite clear that the series defined in (9) is convergent absolutely in $\mathfrak{D}$. Using the definition of $q$-derivative along with the idea of convolutions, we now define the integral operator $\mathfrak{\Im}_{q}^{\mu}: \mathfrak{A} \rightarrow \mathfrak{A}$ by

$$
\Im_{q}^{\mu} f(z)=\mathscr{F}_{q, \mu+1}^{-1}(z) * f(z)=z+\sum_{n=2}^{\infty} \psi_{n-1} a_{n} z^{n},
$$

$(z \in \mathfrak{D})$

with

$$
\psi_{n-1}=\frac{[n, q] !}{[\mu+1, q]_{n-1}}
$$

From (10), we can easily get the identity

$$
\begin{aligned}
{[\mu+1, q] \mathfrak{\Im}_{q}^{\mu} f(z)=} & {[\mu, q] \mathfrak{\Im}_{q}^{\mu+1} f(z) } \\
& +q^{\mu} z \partial_{q}\left(\mathfrak{\Im}_{q}^{\mu+1} f(z)\right) .
\end{aligned}
$$

We note that $\mathfrak{\Im}_{q}^{0} f(z)=z \partial_{q} f(z), \mathfrak{\Im}_{q}^{1} f(z)=f(z)$, and

$$
\lim _{q \rightarrow 1^{-}} \Im_{q}^{\mu} f(z)=z+\sum_{n=2}^{\infty} \frac{n !}{(\mu+1)_{n-1}} a_{n} z^{n}
$$

This shows that, by taking $q \rightarrow 1^{-}$, the operator defined in (10) reduces to the familiar Noor integral operator introduced in $[10,11]$. Also for more details on the $q$-analogue of differential and integral operators, see the work [12-14].

Motivated from the work studied in [7, 15-17], we now define subfamilies of the set $\mathfrak{A}$ by using the operator $\mathfrak{\Im}_{q}^{\mu}$ as follows.

Definition 1. Let $-1 \leq B<A \leq 1$ and $0<q<1$. Then the function $f \in \mathfrak{A}$ is in the class $\mathbb{Q}_{q}(\mu, A, B)$ if it satisfies

$$
\frac{z \partial_{q}\left(\mathfrak{\Im}_{q}^{\mu} f(z)\right)}{\mathfrak{\Im}_{q}^{\mu} f(z)} \prec \frac{1+A z}{1+B z}, \quad(z \in \mathfrak{D}),
$$

where the notion " $\prec$ " denotes the familiar subordinations.
Equivalently, a function $f \in \mathfrak{A}$ is in the class $\mathcal{Q}_{q}(\mu, A, B)$, if and only if

$$
\left|\frac{z \partial_{q}\left(\Im_{q}^{\mu} f(z)\right) / \Im_{q}^{\mu} f(z)-1}{A-B\left(z \partial_{q}\left(\Im_{q}^{\mu} f(z)\right) / \Im_{q}^{\mu} f(z)\right)}\right|<1, \quad(z \in \mathfrak{D}) .
$$

We will assume throughout our discussion, unless otherwise stated, that

$$
\begin{aligned}
\mu & >-1, \\
-1 & \leq B<A \leq 1, \\
0 & <q<1
\end{aligned}
$$

and all coefficients $a_{k}$ are positive.

We need the following result in the proof of a result.

Lemma 2 (see [18]). Let $-1 \leq B_{2} \leq B_{1}<A_{1} \leq A_{2} \leq 1$. Then

$$
\frac{1+A_{1} z}{1+B_{1} z} \prec \frac{1+A_{2} z}{1+B_{2} z} .
$$

\section{Main Results}

Theorem 3. Let $f \in \mathfrak{A}$ be given by (1). Then the function $f$ is in the family $Q_{q}(\mu, A, B)$, if and only if

$$
\sum_{n=2}^{\infty}\{[n, q](1-B)-1+A\} a_{n} \psi_{n-1}<(A-B) .
$$

Proof. Let us assume first that inequality (18) holds. To show $f \in Q_{q}(\mu, A, B)$, we only need to prove the inequality (15). For this, consider

$$
\begin{aligned}
& \left|\frac{z \partial_{q}\left(\Im_{q}^{\mu} f(z)\right) / \Im_{q}^{\mu} f(z)-1}{A-B\left(z \partial_{q}\left(\Im_{q}^{\mu} f(z)\right) / \Im_{q}^{\mu} f(z)\right)}\right| \\
& \quad=\left|\frac{\sum_{n=2}^{\infty} \psi_{n-1}[[n, q]-1] a_{n} z^{n}}{(A-B) z+\sum_{n=2}^{\infty} \psi_{n-1}[A-B[n, q]] a_{n} z^{n}}\right| \\
& \quad \leq \frac{\sum_{n=2}^{\infty} \psi_{n-1}[[n, q]-1] a_{n}}{(A-B)-\sum_{n=2}^{\infty} \psi_{n-1}[A-B[n, q]] a_{n}}<1,
\end{aligned}
$$

where we have used (4), (10), and (18) and this completes the direct part. Conversely, let $f \in Q_{q}(\mu, A, B)$ be of the form (1). Then from (15) along with (10), we have, for $z \in \mathfrak{D}$,

$$
\begin{aligned}
& \left|\frac{z \partial_{q}\left(\mathfrak{\Im}_{q}^{\mu} f(z)\right) / \Im_{q}^{\mu} f(z)-1}{A-B\left(z \partial_{q}\left(\mathfrak{J}_{q}^{\mu} f(z)\right) / \Im_{q}^{\mu} f(z)\right)}\right| \\
& \quad=\left|\frac{\sum_{n=2}^{\infty} \psi_{n-1}[[n, q]-1] a_{n} z^{n}}{(A-B) z+\sum_{n=2}^{\infty} \psi_{n-1}[A-B[n, q]] a_{n} z^{n}}\right|<1 .
\end{aligned}
$$

Since $|\Re \mathfrak{R} z|<|z|$, we have

$$
\operatorname{Re}\left\{\frac{\sum_{n=2}^{\infty} \psi_{n-1}[[n, q]-1] a_{n} z^{n}}{(A-B)+\sum_{n=2}^{\infty} \psi_{n-1}[A-B[n, q]] a_{n} z^{n}}\right\}<1 .
$$


Now we choose values of $z$ on the real axis such that $z \partial_{q}\left(\mathfrak{\Im}_{q}^{\mu} f(z)\right) / \mathfrak{\Im}_{q}^{\mu} f(z)$ is real. Upon clearing the denominator in (21) and letting $z \rightarrow 1^{-}$through real values, we obtain the required inequality (18).

Theorem 4. Let $f \in Q_{q}(\mu, A, B)$. Then

$$
\Im_{q}^{\mu} f(z)=\exp \int_{0}^{z} \frac{1}{t}\left(\frac{1-A \phi(t)}{1-B \phi(t)}\right) d_{q} t,
$$

with $|\phi(z)|<1$ and $z \in \mathfrak{D}$.

Proof. Let $f \in \mathbb{Q}_{q}(\mu, A, B)$ and setting

$$
\frac{z \partial_{q}\left(\mathfrak{\Im}_{q}^{\mu} f(z)\right)}{\mathfrak{J}_{q}^{\mu} f(z)}=v(z),
$$

with

$$
v(z) \prec \frac{1+A z}{1+B z}
$$

equivalently, we can write

$$
\left|\frac{v(z)-1}{A-B v(z)}\right|<1
$$

or in other way, we have

$$
\begin{gathered}
\frac{v(z)-1}{A-B v(z)}=\phi(z), \\
|\phi(z)|<1,
\end{gathered}
$$

$(z \in \mathfrak{D})$

Thus we can rewrite

$$
\frac{z \partial_{q}\left(\mathfrak{J}_{q}^{\mu} f(z)\right)}{\mathfrak{J}_{q}^{\mu} f(z)}=\left(\frac{1-A \phi(t)}{1-B \phi(t)}\right),
$$

and further by simple computation of integration, the proof is completed.

Theorem 5. Let $f_{i} \in Q_{q}(\mu, A, B)$ and have the form

$$
f_{i}(z)=z+\sum_{k=1}^{\infty} a_{k, i} z^{k}, \quad \text { for } i=1,2, \ldots, l .
$$

Then $F \in Q_{q}(\mu, A, B)$, where

$$
F(z)=\sum_{i=1}^{l} c_{i} f_{i}(z) \quad \text { with } \sum_{i=1}^{l} c_{i}=1 .
$$

Proof. By the virtue of Theorem 3, one can write

$$
\sum_{n=2}^{\infty}\left\{\frac{([n, q](1-B)-1+A) \psi_{n-1}}{A-B}\right\} a_{n, i}<1 .
$$

Therefore

$$
\begin{aligned}
F(z) & =\sum_{i=2}^{l} c_{i}\left(z+\sum_{n=2}^{\infty} a_{n, i} z^{n}\right)=z+\sum_{i=2}^{l} \sum_{n=2}^{\infty} c_{i} a_{n, i} z^{n} \\
& =z+\sum_{n=2}^{\infty}\left(\sum_{i=2}^{l} c_{i} a_{n, i}\right) z^{n} ;
\end{aligned}
$$

however

$$
\begin{aligned}
& \sum_{n=2}^{\infty} \frac{([n, q](1-B)-1+A) \psi_{n-1}}{A-B}\left(\sum_{i=2}^{l} a_{n, i} c_{i}\right) \\
& \quad=\sum_{i=2}^{l}\left[\sum_{n=2}^{\infty} \frac{([n, q](1-B)-1+A) \psi_{n-1}}{A-B} a_{n, i}\right] c_{i} \leq 1 ;
\end{aligned}
$$

then $F \in \mathbb{Q}_{q}(\mu, A, B)$. Hence the proof is complete.

Theorem 6. If $f$ and $g$ belong to $\mathbb{Q}_{q}(\mu, A, B)$, then their weighted mean $h_{j}$ is also in $\mathbb{Q}_{q}(\mu, A, B)$, where $h_{j}$ is defined by

$$
h_{j}(z)=\left\{\frac{(1-j) f(z)+(1+j) g(z)}{2}\right\} .
$$

Proof. From (33), we can easily write

$$
h_{j}(z)=z+\sum_{n=2}^{\infty}\left\{\frac{(1-j) a_{n}+(1+j) b_{n}}{2}\right\} z^{n} .
$$

To prove that $h_{j} \in \mathbb{Q}_{q}(\mu, A, B)$, we need to show that

$$
\begin{aligned}
\sum_{n=2}^{\infty}\left\{\frac{[n, q](1-B)-1+A}{A-B}\right\} \\
\cdot\left\{\frac{(1-j) a_{n}+(1+j) b_{n}}{2}\right\} \psi_{n-1}<1 .
\end{aligned}
$$

For this, consider

$$
\begin{aligned}
& \sum_{n=2}^{\infty}\left\{\frac{[n, q](1-B)-1+A}{A-B}\right\} \\
& \quad \cdot\left\{\frac{(1-j) a_{n}+(1+j) b_{n}}{2}\right\} \psi_{n-1}=\frac{(1-j)}{2} \\
& \quad \cdot \sum_{n=2}^{\infty}\left\{\frac{[n, q](1-B)-1+A}{A-B}\right\} \psi_{n-1} a_{n}+\frac{(1+j)}{2} \\
& \quad \cdot \sum_{n=2}^{\infty}\left\{\frac{[n, q](1-B)-1+A}{A-B}\right\} \psi_{n-1} b_{n}<\frac{(1-j)}{2} \\
& +\frac{(1+j)}{2}=1,
\end{aligned}
$$

where we have used inequality (18). Hence the result follows. 
Theorem 7. Let $f_{i}$ with $i=1,2, \ldots, \lambda$ belong to the class $Q_{q}(\mu, A, B)$. Then the arithmetic mean $h$ of $f_{i}$ is given by

$$
h(z)=\frac{1}{\lambda} \sum_{i=1}^{\lambda} f_{i}(z)
$$

and is also in the class $\mathbb{Q}_{q}(\mu, A, B)$.

Proof. From (37), we can write

$$
h(z)=\frac{1}{\lambda} \sum_{i=1}^{\lambda}\left(z+\sum_{n=2}^{\infty} a_{n, i} z^{n}\right)=z+\sum_{n=2}^{\infty}\left(\frac{1}{\lambda} \sum_{i=1}^{\lambda} a_{n, i}\right) z^{n} .
$$

Since $f_{i} \in Q_{q}(\mu, A, B)$ for every $i=1,2, \ldots, \lambda$, using (38) and (18), we have

$$
\begin{aligned}
& \sum_{n=2}^{\infty} \psi_{n-1}\{[n, q](1-B)-1+A\}\left(\frac{1}{\lambda} \sum_{i=1}^{\lambda} a_{n, i}\right) \\
& =\frac{1}{\lambda} \sum_{i=1}^{\lambda}\left(\sum_{n=2}^{\infty} \psi_{n-1}\{[n, q](1-B)-1+A\} a_{n, i}\right) \\
& \leq \frac{1}{\lambda} \sum_{i=1}^{\lambda}(A-B)=(A-B),
\end{aligned}
$$

and this completes the proof.

Theorem 8. Let $f \in \mathbb{Q}_{q}(\mu, A, B)$. Then $f$ is in the family $\mathcal{S}^{*}(\beta)$ of starlike functions of order $\beta(0 \leq \beta<1)$ for $|z|<r_{1}$, where

$$
r_{1}^{r_{1}}=\left(\frac{(1-\beta)([n, q](1-B)-1+A)[n, q] !}{(n-\beta)(A-B)[\mu+1, q]_{n-1}}\right)^{1 /(n-1)} .
$$

Proof. Let $f \in Q_{q}(\mu, A, B)$. To prove $f \in \mathcal{S}^{*}(\beta)$, we only need to show

$$
\left|\frac{z f^{\prime}(z) / f(z)-1}{z f^{\prime}(z) / f(z)+1-2 \beta}\right|<1 .
$$

Using (1) along with some simple computation yields

$$
\sum_{n=2}^{\infty}\left(\frac{n-\beta}{1-\beta}\right)\left|a_{n}\right||z|^{n-1}<1 .
$$

Since $f \in \mathbb{Q}_{q}(\mu, A, B)$, from (18), we can easily obtain

$$
\sum_{n=2}^{\infty} \frac{[n, q] !}{[\mu+1, q]_{n-1}}\left(\frac{[n, q](1-B)-1+A}{(A-B)}\right)\left|a_{n}\right|<1 .
$$

Now inequality (42) will be true, if the following holds:

$$
\begin{aligned}
& \sum_{n=2}^{\infty}\left(\frac{n-\beta}{1-\beta}\right)\left|a_{n}\right||z|^{n-1} \\
& \quad<\sum_{n=2}^{\infty} \frac{[n, q] !}{[\mu+1, q]_{n-1}}\left(\frac{[n, q](1-B)-(1-A)}{A-B}\right)\left|a_{n}\right|,
\end{aligned}
$$

which implies that

$$
|z|^{n-1}<\frac{(1-\beta)([n, q](1-B)-1+A)[n, q] !}{(n-\beta)(A-B)[\mu+1, q]_{n-1}},
$$

and thus we get the needed result.

Theorem 9. Let $-1 \leq B_{2} \leq B_{1}<A_{1} \leq A_{2} \leq 1$ and $\mathfrak{\Im}_{q}^{\mu+1} f(z) \neq 0$ in $\mathfrak{D}$, and this satisfies

$$
\frac{[\mu+1, q]}{q^{\mu}}\left\{\frac{\mathfrak{\Im}_{q}^{\mu} f(z)}{\mathfrak{\Im}_{q}^{\mu+1} f(z)}-\frac{[\mu, q]}{[\mu+1, q]}\right\} \prec \frac{1+A_{1} z}{1+B_{1} z} .
$$

Then $f \in \mathbb{Q}_{q}\left(\mu+1, A_{2}, B_{2}\right)$.

Proof. Since $\mathfrak{\Im}_{q}^{\mu+1} f(z) \neq 0$ in $\mathfrak{D}$, therefore let us define the function $p(z)$ by

$$
\frac{z \partial_{q}\left(\mathfrak{\Im}_{q}^{\mu+1} f(z)\right)}{\mathfrak{J}_{q}^{\mu+1} f(z)}=p(z) \quad(z \in \mathfrak{D}) .
$$

By the virtue of identity (12), we obtain

$$
\frac{1}{q^{\mu}}\left\{[\mu+1, q] \frac{\mathfrak{\Im}_{q}^{\mu} f(z)}{\mathfrak{\Im}_{q}^{\mu+1} f(z)}-[\mu, q]\right\}=p(z) .
$$

Therefore, using (46), we have

$$
\frac{z \partial_{q}\left(\mathfrak{\Im}_{q}^{\mu+1} f(z)\right)}{\mathfrak{\Im}_{q}^{\mu+1} f(z)}=p(z) \prec \frac{1+A_{1} z}{1+B_{1} z},
$$

and now, using Lemma 2 , we have $f \in \mathbb{Q}_{q}\left(\mu+1, A_{2}, B_{2}\right)$.

\section{Conflicts of Interest}

The authors agree with the contents of the manuscript and there are no conflicts of interest among the authors.

\section{Acknowledgments}

This work is supported by National Natural Science Foundation of China (Grant no. 11571299) and Natural Science Foundation of Jiangsu Province (Grant no. BK20151304).

\section{References}

[1] F. H. Jackson, "On $q$-functions and a certain difference operator," Earth and Environmental Science Transactions of the Royal Society of Edinburgh, vol. 46, no. 2, pp. 253-281, 1909.

[2] F. H. Jackson, "On $q$-definite integrals," The Quarterly J. Pure Appl. Math, vol. 41, pp. 193-203, 1910.

[3] A. Aral and V. Gupta, "Generalized q-Baskakov operators," Mathematica Slovaca, vol. 61, no. 4, pp. 619-634, 2011.

[4] A. Aral and V. Gupta, "On $q$-Baskakov type operators," Demonstratio Mathematica, vol. 42, no. 1, pp. 109-122, 2009.

[5] G. A. Anastassiou and S. G. Gal, "Geometric and approximation properties of generalized singular integrals in the unit disk," Journal of the Korean Mathematical Society, vol. 23, no. 2, pp. 425-443, 2006. 
[6] A. Aral, "On the generalized Picard and Gauss Weierstrass singular integrals," Journal of Computational Analysis and Applications, vol. 8, no. 3, pp. 249-261, 2006.

[7] S. a. Kanas and D. Raducanu, "Some class of analytic functions related to conic domains," Mathematica Slovaca, vol. 64, no. 5, pp. 1183-1196, 2014.

[8] H. Aldweby and M. Darus, "Some subordination results on $q$-analogue of Ruscheweyh differential operator," Abstract and Applied Analysis, vol. 2014, Article ID 958563, 2014.

[9] S. Mahmood and J. Sokół, "New subclass of analytic functions in conical domain associated with Ruscheweyh $q$-differential operator," Results in Mathematics, vol. 71, pp. 1-13, 2017.

[10] K. I. Noor, "On new classes of integral operators," Journal of Natural Geometry, vol. 16, no. 1-2, pp. 71-80, 1999.

[11] K. I. Noor and M. A. Noor, "On integral operators," Journal of Mathematical Analysis and Applications, vol. 238, no. 2, pp. 341352, 1999.

[12] I. Aldawish and M. Darus, "Starlikeness of q-differential operator involving quantum calculus," Korean Journal of Mathematics, vol. 22, no. 4, pp. 699-709, 2014.

[13] H. Aldweby and M. Darus, "A subclass of harmonic univalent functions associated with $q$-analogue of Dziok-Srivastava operator," ISRN Mathematical Analysis, vol. 2013, Article ID 382312, 6 pages, 2013.

[14] A. Mohammed and M. Darus, "A generalized operator involving the q-hypergeometric function," Matematicki Vesnik, vol. 65, no. 4, pp. 454-465, 2013.

[15] S. Agrawal and S. K. Sahoo, "A generalization of starlike functions of order alpha," Hokkaido Mathematical Journal, vol. 46, no. 1, pp. 15-27, 2017.

[16] H. Aldweby and M. Darus, "Integral operator defined by $q$ analogue of Liu-Srivastava operator," Studia. Universitatis BabeBolyai Mathematica, vol. 58, no. 4, pp. 529-537, 2013.

[17] T. M. Seoudy and M. K. Aouf, "Coefficient estimates of new classes of $q$-starlike and q-convex functions of complex order," Journal of Mathematical Inequalities, vol. 10, no. 1, pp. 135-145, 2016.

[18] M. S. Liu, "On a subclass of p-valent close to convex functions of type $\alpha$ and order $\beta$," Journal of Mathematical Study, vol. 30, no. 1, pp. 102-104. 


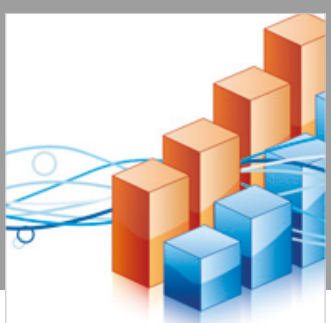

Advances in

Operations Research

\section{-n-m}
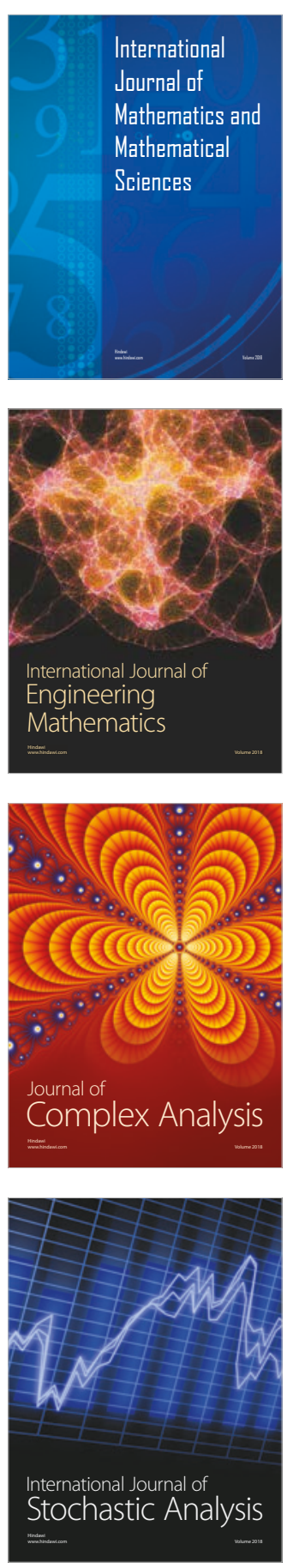
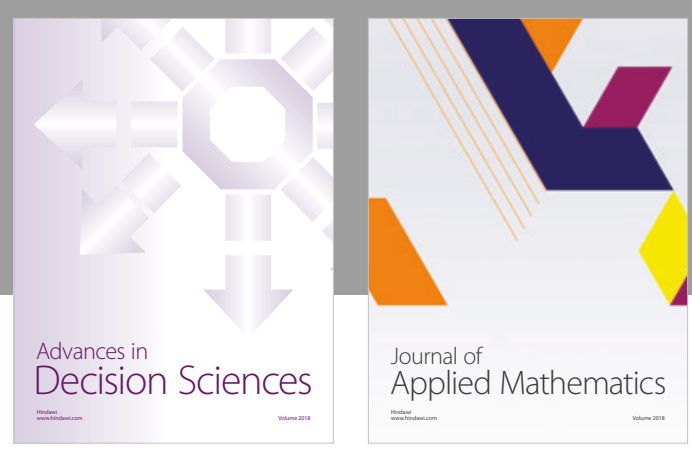

Journal of

Applied Mathematics
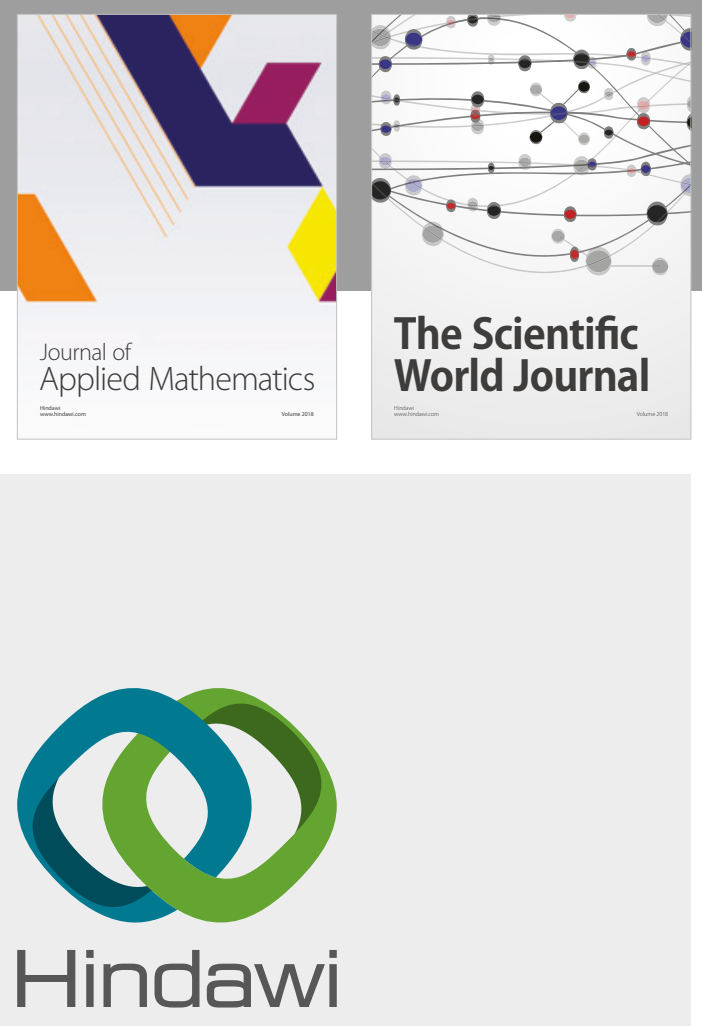

Submit your manuscripts at

www.hindawi.com

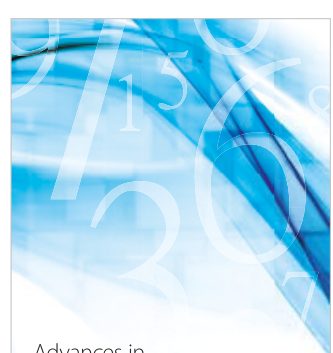

Advances in
Numerical Analysis
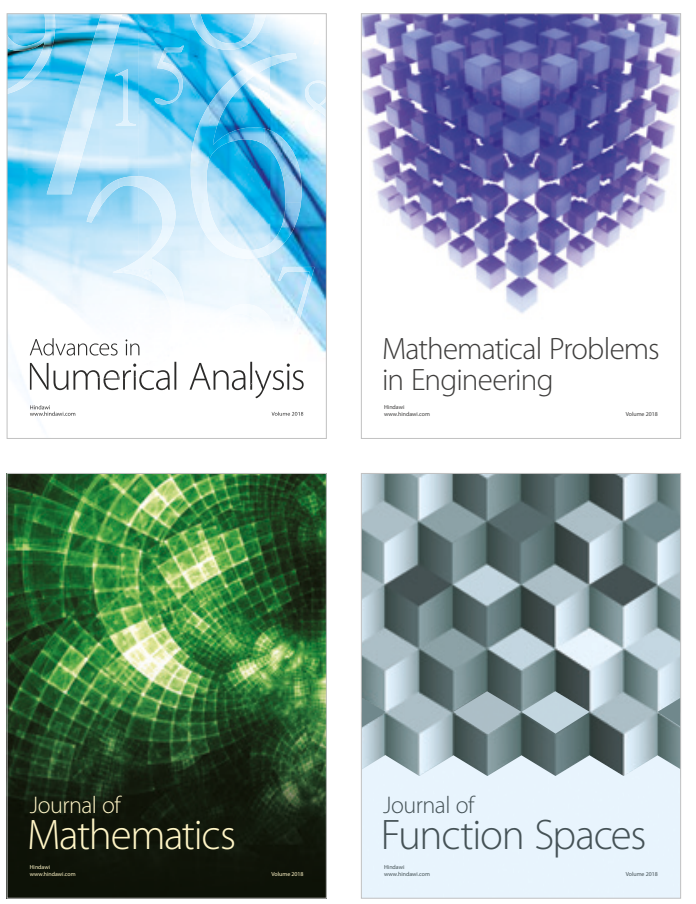

Mathematical Problems in Engineering

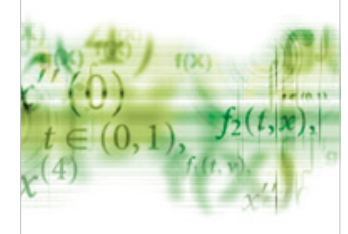

International Journal of

Differential Equations

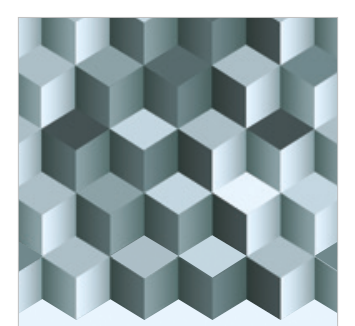

Journal of

Function Spaces

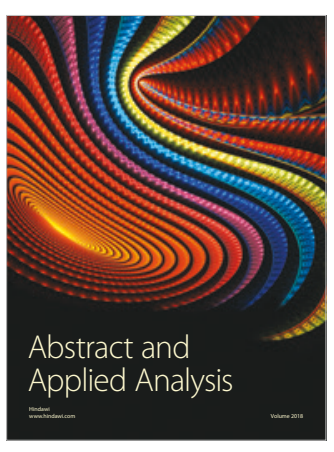

The Scientific

World Journal

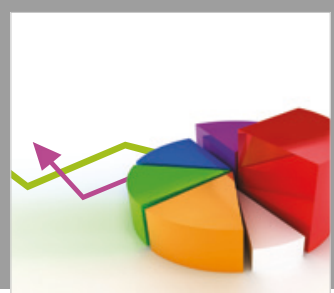

Journal of

Probability and Statistics
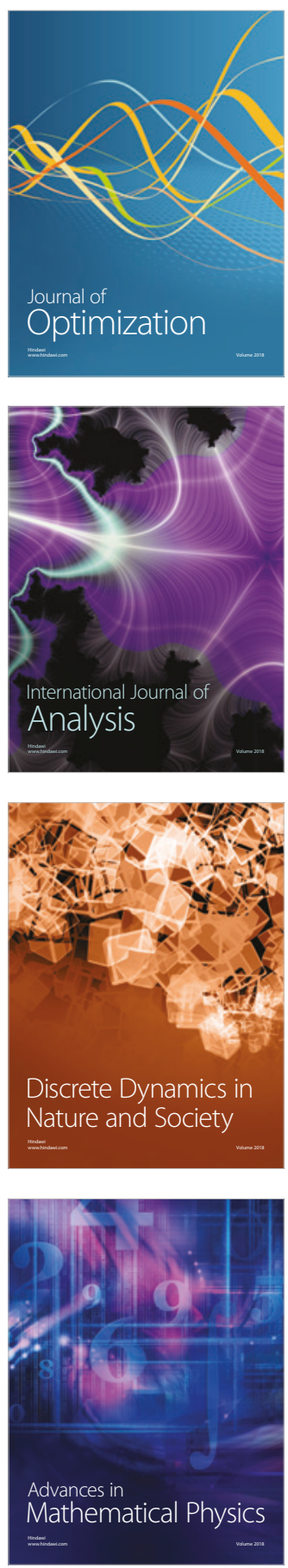